\title{
A POD-based reduced-order TSCFE extrapolation iterative format for two-dimensional heat equations
}

Zhendong Luo*

\section{${ }^{*}$ Correspondence:}

zhdluo@ncepu.edu.cn

School of Mathematics and Physics,

North China Electric Power

University, No. 2 Bei Nong Road,

Beijing, 102206, China

\begin{abstract}
In this article, a proper orthogonal decomposition (POD) technique is employed to establish a POD-based reduced-order time-space continuous finite element (TSCFE) extrapolation iterative format for two-dimensional (2D) heat equations, which includes very few degrees of freedom but holds sufficiently high accuracy. The error estimates of the POD-based reduced-order TSCFE solutions and the algorithm implementation of the POD-based reduced-order TSCFE extrapolation iterative format are provided. A numerical example is used to illustrate that the results of the numerical computation are consistent with the theoretical conclusions. Moreover, it is shown that the POD-based reduced-order TSCFE extrapolation iterative format is feasible and efficient for solving $2 \mathrm{D}$ heat equations.
\end{abstract}

MSC: 74S10; 65M15; 35Q35

Keywords: proper orthogonal decomposition technique; reduced-order time-space finite element extrapolation iterative format; two-dimensional heat equations; error estimate

\section{Introduction}

The time-space finite element (FE) methods for time-dependent partial differential equations (TDPDEs) play an important role in many practical applications and form an important research topic (see [1-7]). They have a higher accuracy than their usual FE methods with time-forward difference and even they have a higher accuracy than their CrankNicolson FE methods with time-averaged data (see, e.g., [8-10]). However, even if the time-space continuous finite element (TSCFE) methods for two-dimensional (2D) heat equations include a lot of degrees of freedom too, they would cause many difficulties for real-life applications. Therefore, an important problem is how as few it is possible to use to lessen the degrees of freedom so as to alleviate the computational load and save timeconsuming calculations and resource demands in the computational process in a way that guarantees the sufficiently accurate numerical solutions.

The proper orthogonal decomposition (POD) method (see [11]) is an efficient means to lessen the degrees of freedom of numerical models for TDPDEs and alleviate the accumulation of truncation errors in the computational process so as to reduce the computational load and save memory requirements. It has been widely and successfully applied to numerous fields, including signal analysis and pattern recognition (see [12]), statistics,

( 2015 Luo; licensee Springer. This is an Open Access article distributed under the terms of the Creative Commons Attribution License (http://creativecommons.org/licenses/by/4.0), which permits unrestricted use, distribution, and reproduction in any medium, provided the original work is properly credited. 
geophysical fluid dynamics or meteorology (see [13]). It essentially provides an orthogonal basis for representing the given data in a certain least squares optimal sense, namely, it provides a way to find optimal lower dimensional approximations of the given data. Especially, it has played an important role in reduced-order of numerical methods for TDPDEs (see, e.g., [14-31]). Moreover, the long-term stability of POD reduced-order models is discussed (see, e.g., [32-35]).

However, almost all existing POD-based reduced-order numerical methods (see, e.g., [14-35]) employ numerical solutions obtained from classical numerical methods on the total time span $[0, T]$ to form POD bases and establish reduced-order models, and then recompute the solutions on the same time span $[0, T]$, which actually entails repeating computations on the same time span $[0, T]$. Especially, to the best of our knowledge, there is not any report that the POD-based reduced-order TSCFE extrapolation iterative format for 2D heat equations is established or that an algorithm of the reduced-order TSCFE extrapolation iterative format is implemented. Therefore, in this article, we establish the POD-based reduced-order TSCFE extrapolation iterative format for 2D heat equations and provide the error estimates of the POD-based reduced-order TSCFE solutions and the algorithm implementation of the POD-based reduced-order TSCFE extrapolation iterative format. We also provide a numerical example to illustrate that the POD-based reduced-order TSCFE extrapolation iterative format is feasible and efficient for seeking numerical solutions for 2D heat equations. Especially, we here thoroughly improve the existing methods, namely, we do only employ the first few given classical TSCFE solutions on a very short time span $\left[0, T_{0}\right]\left(T_{0} \ll T\right)$ as snapshots to form POD basis and establish the POD-based reduced-order TSCFE extrapolation iterative format for seeking the numerical solutions on the total time span $[0, T]$. Thus, we can sufficiently absorb the advantage of the POD method, namely, sufficiently utilize the given data (on very short time span $\left[0, T_{0}\right]$ and $T_{0} \ll T$ ) to forecast future physical phenomena (on the time span $\left.\left[T_{0}, T\right]\right)$. Therefore, the POD-based reduced-order TSCFE extrapolation iterative format for 2D heat equations is completely different from the existing POD-based reduced-order methods (see, e.g., [14-35] etc.) and we have an improvement and development of the existing methods as mentioned above or others.

The article is organized as follows. Section 2 recalls the classical TSCFE method for 2D heat equations and generates snapshots by means of the first fewer TSCFE solutions obtained from the classical TSCFE formulation. In Section 3, we form orthonormal POD bases of a set of the snapshots by means of the POD method and establish the POD-based reduced-order TSCFE extrapolation iterative format including very few degrees of freedom but holding sufficiently high accuracy for the 2D heat equations. In Section 4, the error estimates of the POD-based reduced-order TSCFE solutions and the algorithm implementation for the POD-based reduced-order TSCFE extrapolation iterative format are provided. In Section 5, a numerical example is presented to illustrate that the results of the numerical computation are consistent with the theoretical conclusions and validate that the POD-based reduced-order TSCFE extrapolation iterative format is feasible and efficient for finding numerical solutions to the $2 \mathrm{D}$ heat equations and can greatly lessen its degrees of freedom and alleviate the computational load as well as save time for calculations and resource demands in the computational process. Section 6 provides the main conclusions and discussions. 


\section{Recall the classical TSCFE method for 2D heat equations and generate snapshots}

Let $\Omega \subset \mathbf{R}^{2}$ be a bounded and connected polygonal domain. Consider the following 2D heat equations (see $[11,12]$ ).

Problem I Find $u$ such that

$$
\begin{aligned}
& u_{t}-\gamma \Delta u=f, \quad(x, y, t) \in \Omega \times(0, T] \\
& u(x, y, t)=0, \quad(x, y, t) \in \partial \Omega \times(0, T], \\
& u(x, y, 0)=\varphi_{0}(x, y), \quad(x, y) \in \Omega,
\end{aligned}
$$

where $u_{t}=\partial u / \partial t, \gamma$ is a positive constant, the source term $f(x, y, t)$ and the initial value function $\varphi_{0}(x, y)$ are all sufficiently smooth to ensure the following analysis' validity, and $T$ is the total time.

The Sobolev spaces along with their properties used in this context are standard (see [36]). For example, define the space

$$
H^{m}(\Omega)=\left\{v ; \sum_{0 \leq|\alpha| \leq m} \int_{\Omega}\left|\frac{\partial^{|\alpha|} v}{\partial x^{\alpha_{1}} \partial y^{\alpha_{2}}}\right|_{0}^{2} \mathrm{~d} x \mathrm{~d} y<\infty\right\},
$$

where $m \geq 0$ is integer, $\alpha=\left(\alpha_{1}, \alpha_{2}\right), \alpha_{1}$, and $\alpha_{2}$ are two non-negative integers, and $|\alpha|=$ $\alpha_{1}+\alpha_{2}$, with norm

$$
\|v\|_{m}=\left[\sum_{0 \leq|\alpha| \leq m} \int_{\Omega}\left|\frac{\partial^{|\alpha|} v}{\partial x^{\alpha_{1}} \partial y^{\alpha_{2}}}\right|_{0}^{2} \mathrm{~d} x \mathrm{~d} y\right]^{1 / 2}
$$

and semi-norm

$$
|v|_{m}=\left[\sum_{|\alpha|=m} \int_{\Omega}\left|\frac{\partial^{|\alpha|} v}{\partial x^{\alpha_{1}} \partial y^{\alpha_{2}}}\right|_{0}^{2} \mathrm{~d} x \mathrm{~d} y\right]^{1 / 2} .
$$

Time-space Sobolev spaces are denoted by

$$
H^{l}\left(0, t_{n} ; H^{m}(\Omega)\right)=\left\{v(x, t) ; \int_{0}^{t_{n}} \sum_{i=0}^{l} \frac{\mathrm{d}^{i}}{\mathrm{~d} t^{i}}\|v(\cdot, t)\|_{m}^{2} \mathrm{~d} t<\infty\right\}
$$

with norms

$$
\|v\|_{H^{l}\left(0, t_{n} ; H^{m}\right)}=\left[\int_{0}^{t_{n}} \sum_{i=0}^{l} \frac{\mathrm{d}^{i}}{\mathrm{~d} t^{i}}\|v(\cdot, t)\|_{m}^{2} \mathrm{~d} t\right]^{1 / 2} .
$$

Especially, $\|v\|_{H^{l}\left(0, t_{n} ; H^{m}\right)}$ are denoted by $\|v\|_{H^{l}\left(H^{m}\right)}$ if $t_{n}=T . H_{0}^{1}(\Omega)=\left\{v \in H^{1}(\Omega) ;\left.v\right|_{\partial \Omega}=0\right\}$. Let $U=H^{1}\left(0, T ; H_{0}^{1}(\Omega)\right)$. Thus, a variational formulation for Problem I is written as follows. 
Problem II Seek $u(t) \in U$ satisfying

$$
\begin{aligned}
& \left(u_{t}, v\right)+a(u, v)=(f, v), \quad \forall v \in U, \\
& u(x, y, 0)=\varphi_{0}(x, y), \quad(x, y) \in \Omega,
\end{aligned}
$$

where $a(u, v)=\gamma(\nabla u, \nabla v),(\cdot, \cdot)$ denotes inner product in $L^{2}(\Omega)$. For the sake of convenience and without loss of generality, we may as well suppose that $\gamma=1$ in the following theoretical analysis.

In order to establish TSCFE formulation, we first subdivide computational domain $\bar{\Omega}$ into a quasi-uniform triangulation $\Im_{h}=\{K\}$ with $h=\max h_{K}$, here $h_{K}$ is the diameter of the triangle $K \in \Im_{h}$ (see $[8,37,38]$ ), and take the partition on the time span $[0, T]$ as $0=t_{0}<t_{1}<\cdots<t_{N}=T$ with a time step $k=\max _{1 \leq i \leq N}\left|t_{i}-t_{i-1}\right|$. Then we introduce the subspace $S_{h m}(\Omega) \subset H_{0}^{1}(\Omega)$ consisting of the piecewise continuous $m$ th degree polynomials defined on the partition $\Im_{h}$ of $\Omega$ with mesh parameter $h$, and let $S_{k l}(0, T)$ be an FE subspace on time partition consisting of continuous piecewise $l$ th degree polynomials, namely, $S_{k l}(0, T)=\left\{\chi \in C^{0}([0, T]):\left.\chi\right|_{\left[t_{i-1}, t_{i}\right]} \in P_{l}\left(\left[t_{i-1}, t_{i}\right]\right)\right\}$, where $P_{l}\left(\left[t_{i-1}, t_{i}\right]\right)$ is the set of polynomials of degree $\leq l$ on $\left[t_{i-1}, t_{i}\right]$. Finally, we define the time-space element subspace as

$$
U_{h k}=S_{h m}(\Omega) \otimes S_{k l}(0, T) .
$$

Thus, the classical TSCFE formulation for the 2D heat equations is established as follows.

Problem III Find $u^{h k} \in U_{h k}$ such that

$$
\begin{aligned}
& \int_{0}^{T}\left[\left(u_{t}^{h k}, \phi_{t}\right)+a\left(u^{h k}, \phi_{t}\right)\right] \mathrm{d} t=\int_{0}^{T}\left(f, \phi_{t}\right) \mathrm{d} t, \quad \forall \phi \in U_{h k}, \\
& u^{h k}(x, y, 0)=P_{h} \varphi_{0}(x, y), \quad(x, y) \in \Omega,
\end{aligned}
$$

where $P_{h}: H_{0}^{1}(\Omega) \rightarrow S_{h m}(\Omega)$ is a Ritz projection, namely, for $u \in H_{0}^{1}(\Omega)$, we have

$$
\left(\nabla P_{h} u, \nabla \phi\right)=(\nabla u, \nabla \phi), \quad \forall \phi \in S_{h m}(\Omega) .
$$

The TSCFE solution $u^{h k}$ can be found by marching through successive time levels. To this end, let $J^{n}=\left[t_{n-1}, t_{n}\right]$ and $P_{l}\left(J^{n}\right)$ denote the set of polynomials of degree $\leq l$ on an interval $J^{n}$. Then, for $n=1,2, \ldots, N$, the TSCFE solution $u^{h k}$ on $J^{n}$ is found as the unique solution of

$$
\int_{J^{n}}\left[\left(u_{t}^{h k}, \phi\right)+a\left(u^{h k}, \phi\right)\right] \mathrm{d} t=\int_{J^{n}}(f, \phi) \mathrm{d} t, \quad \forall \phi \in S_{h m}(\Omega) \otimes P_{l-1}\left(J_{n}\right),
$$

with $u^{h k}(x, y, 0)=P_{h} \varphi_{0}(x, y)$, and $u^{h k}\left(x, y, t_{n}\right)(n=1,2, \ldots, N-1,(x, y) \in \Omega)$ are given and have been found on a previous time step.

In order to improve the accuracy of time approximation, $P_{l-1}\left(J^{n}\right)$ is taken as the set of Gauss-Legendre polynomials with accuracy for all polynomials of degree $\leq 2 l-1$. To this 
end, for each $l \geq 1$, consider the Gauss-Legendre integration rule,

$$
\int_{0}^{1} g(\tau) \mathrm{d} \tau \cong \sum_{j=1}^{l} w_{j} g\left(\tau_{j}\right), \quad 0<\tau_{1}<\tau_{2}<\cdots<\tau_{l}<1,
$$

which is exact for all polynomials of degree $\leq 2 l-1$. For given $l \geq 1$, let $\left\{\ell_{i}\right\}_{i=0}^{l}$ be the Lagrange polynomials of degree $l-1$ corresponding to the points of division $\tau_{1}, \tau_{2}, \ldots, \tau_{l}$, namely

$$
\ell_{i}(\tau)=\prod_{\substack{j=1 \\ j \neq i}}^{l} \frac{\tau-\tau_{j}}{\tau_{i}-\tau_{j}}, \quad i=1,2, \ldots, l .
$$

With the linear transformation $t=t_{n}+\tau k_{n}\left(k_{n}=t_{n}-t_{n-1}\right)$ which maps the unit interval $[0,1]$ onto $J^{n}$ and quadrature formula (2.10), the points of division and weights are denoted by

$$
\begin{aligned}
& t_{n, j}=t_{n}+\tau_{j} k_{n}, \quad \ell_{n, j}(t)=\ell_{j}(\tau), \\
& w_{n, j}=\int_{J^{n}} \ell_{n, j}(t) \mathrm{d} t=k_{n} \int_{0}^{1} \ell_{j}(\tau) \mathrm{d} \tau=k_{n} w_{j}, \quad j=1,2, \ldots, l .
\end{aligned}
$$

The Lagrange polynomials $\left\{\tilde{\ell}_{i}\right\}_{i=0}^{l}$ of degree $l$ corresponding to the points of division $0=$ $\tau_{0}<\tau_{1}<\tau_{2}<\cdots<\tau_{l}=1$ are denoted by

$$
\tilde{\ell}_{i}(\tau)=\prod_{\substack{j=0 \\ j \neq i}}^{l} \frac{\tau-\tau_{j}}{\tau_{i}-\tau_{j}}, \quad i=0,1, \ldots, l .
$$

Put $t_{n, 0}=t_{n}$. Note that $u^{n, 0}=u^{h k}\left(x, y, t_{n}\right)(n=0,1,2, \ldots, N-1)$ and if $\psi(x, y) \in S_{h m}(\Omega)$, function $\varphi=\psi(x, y) \ell_{n, i}(t) \in S_{h m}(\Omega) \otimes S_{k(l-1)}(0, T)$. Then $\left.u^{h k}\right|_{j^{n}}$ is solely determined by the functions $u^{n, j}(x, y) \equiv u^{h k}\left(x, y, t_{n, j}\right) \in S_{h m}(\Omega)$ such that

$$
\begin{aligned}
& \sum_{j=1}^{l} m_{i j}\left(u^{n, j}, \psi\right)+k_{n} w_{i}\left(\nabla u^{n, i}, \nabla \psi\right) \\
& =\int_{J^{n}} \ell_{n, i}(f(x, y, t), \psi) \mathrm{d} t, \quad i=1,2, \ldots, l, \forall \psi \in S_{h m}(\Omega), \\
& u^{h k}(x, y, t)=\sum_{j=0}^{l} \tilde{\ell}_{n, j}(t) u^{n, j}(x, y), \quad t \in J^{n}, n=1,2, \ldots, N,
\end{aligned}
$$

where $m_{i, j}=\int_{J^{n}} \tilde{\ell}_{n, j}^{\prime}(t) \ell_{n, i}(t) \mathrm{d} t(i=1,2, \ldots, l ; j=0,1, \ldots, l)$.

The following results of the existence, the uniqueness, the stability, and the convergence of the solution for the system of equations (2.14) and (2.15), namely, Problem III, are obtained by means of the same methods as the proofs of Theorems 3.1, 3.2, 3.3, and 4.4 in [1] or the analogous approaches of proving Theorems 3.1, 4.1, 4.2, and 5.1 in [2].

Theorem 2.1 If $\varphi_{0}(x, y) \in H_{0}^{1}(\Omega)$ and $f(x, y, t) \in L^{2}\left(0, T ; L^{2}(\Omega)\right)$, there exists a unique solution $u^{\text {hk }}$ for the system of equations (2.14) and (2.15), namely, Problem III satisfying

$$
\left\|u_{t}^{h k}\right\|_{L^{2}\left(0, t_{n} ; H^{1}\right)}+\left\|\nabla u^{h k}\left(x, y, t_{n}\right)\right\|_{0} \leq C\left(\|f\|_{L^{2}\left(0, t_{n} ; H^{-1}\right)}+\left\|\nabla \varphi_{0}\right\|_{0}\right) ;
$$


$C$ here and in the following is a constant which is possibly different at different occurrences, being independent of $h$ and $k$, but dependent on $\gamma$.

(1) Let $u(x, y, t)$ of the solution to Problem II satisfy $u(x, y, t) \in H^{m+1}(\Omega)(0 \leq t \leq T), \Delta u \in$ $H^{l+1}\left(0, T ; L^{2}(\Omega)\right)$, and $u \in H^{1}\left(0, T ; H^{m}(\Omega)\right)$. Then we have the following error estimates:

$$
\begin{aligned}
& \left\|u\left(x, y, t_{n}\right)-u^{h k}\left(x, y, t_{n}\right)\right\|_{1} \\
& \leq C\left[k^{l+1}\|u\|_{H^{l+1}\left(0, t_{n} ; H^{2}\right)}+h^{m}\left(\left\|u_{t}\right\|_{L^{2}\left(0, t_{n} ; H^{m}\right)}+\left\|u\left(x, y, t_{n}\right)\right\|_{m+1}\right)\right], \\
& \quad n=1,2, \ldots, N \\
& \left\|u_{t}(x, y, t)-u_{t}^{h k}(x, y, t)\right\|_{L^{2}\left(0, t_{n} ; L^{2}\right)} \\
& \leq C\left\{h^{m+1}\left\|u_{t}\right\|_{L^{2}\left(0, t_{n} ; H^{m+1}\right)}+k^{l}\left[\|u\|_{H^{l+1}\left(0, t_{n} ; L^{2}\right)}+\|u\|_{H^{l}\left(0, t_{n} ; H^{2}\right)}\right]\right\} \\
& \quad n=1,2, \ldots, N .
\end{aligned}
$$

(2) If $\Omega$ is convex and the solution $u$ to Problem II is sufficiently smooth that $u \in$ $H^{l+1}\left(0, T ; H^{1}(\Omega)\right), u \in H^{1}\left(0, T ; H^{m+1}(\Omega)\right)$, and $u(x, y, t) \in H^{m+1}(\Omega)$ for each $t \in[0, T]$, then we have the following error estimates:

$$
\begin{aligned}
& \left\|u\left(x, y, t_{n}\right)-u^{h k}\left(x, y, t_{n}\right)\right\|_{0} \\
& \leq C\left\{k^{l+1}\|u\|_{H^{l+1}\left(0, t_{n} ; H^{1}\right)}+h^{m+1}\left[\left\|u\left(x, y, t_{n}\right)\right\|_{m+1}+\|u\|_{H^{1}\left(0, t_{n} ; H^{m+1}\right)}\right]\right\} \\
& \quad n=1,2, \ldots, N .
\end{aligned}
$$

Remark 2.2 If the source term $f(x, y, t)$, the initial value function $\varphi_{0}(x, y)$, the time step $k$, and the spatial mesh size $h$ all are given, then we can obtain solutions $u^{h k}(x, y, t)$ by solving Problem III or the system of equations (2.14) and (2.15). But we obtain the first $M$ solutions $u^{n, 0}(x, y)=u\left(x, y, t_{n}\right)(1 \leq n \leq M)$ (in general, $M \ll N$, for example, $M=20$, $N=1,000)$ by solving $(2.15)$ at $n=1,2, \ldots, M$, which are referred to as snapshots. When one computes actual problems, one may obtain the ensemble of snapshots from physical system trajectories by drawing samples from experiments.

\section{Form POD bases and establish POD-based reduced-order TSCFE extrapolation iterative format}

In this section, we refer to the idea in $[15,24,26,27]$ to form a POD basis (for more details see $[15,24,26,27])$ and establish the POD-based reduced-order TSCFE extrapolation iterative format for $2 \mathrm{D}$ heat equations.

Form the matrix $\mathbf{A}=\left(A_{i j}\right)_{M \times M} \in R^{M \times M}$, where $A_{i j}=\left(\nabla u^{i, 0}, \nabla u^{j, 0}\right) / M$ and $u^{n, 0}(x, y)(n=$ $1,2, \ldots, M)$ are the snapshots extracted in Section 2. Since the matrix $\mathbf{A}$ is positive semidefinite with $\operatorname{rank} \kappa=\operatorname{dim}\left(\operatorname{span}\left\{u^{n, 0}(x, y) ; n=1,2, \ldots, M\right\}\right)$, its positive eigenvalues and the corresponding orthonormal eigenvectors are used to form POD bases as follows [15, 24, $26,27]$.

Lemma 3.1 Suppose $\lambda_{1} \geq \lambda_{2} \geq \cdots \geq \lambda_{\kappa}>0$ denote the positive eigenvalues of $\mathbf{A}$ and $\mathbf{v}^{i}=$ $\left(v_{1}^{i}, v_{2}^{i}, \ldots, v_{M}^{i}\right)^{T}(i=1,2, \ldots, \kappa)$ the corresponding orthonormal eigenvectors. Then a set of 
POD bases is denoted by

$$
\psi_{i}(x, y)=\frac{1}{\sqrt{M \lambda_{i}}} \sum_{j=1}^{M} v_{j}^{i} u^{j, 0}(x, y), \quad 1 \leq i \leq d \leq \kappa .
$$

Furthermore, there is the following error formula:

$$
\frac{1}{M} \sum_{i=1}^{M}\left|u^{i, 0}-\sum_{j=1}^{d}\left(\nabla u^{i, 0}, \nabla \psi_{j}\right) \psi_{j}\right|_{1}^{2}=\sum_{j=d+1}^{\kappa} \lambda_{j}
$$

Let $S_{d m}(\Omega)=\operatorname{span}\left\{\psi_{1}, \psi_{2}, \ldots, \psi_{d}\right\}$. For $u^{n, 0}(x, y) \in S_{h m}(\Omega)$, define the projection $P^{d}$ : $S_{h m}(\Omega) \rightarrow S_{d m}(\Omega)$ as follows:

$$
\left(\nabla P^{d} u^{n, 0}, \nabla w_{d}\right)=\left(\nabla u^{n, 0}, w_{d}\right), \quad \forall w_{d} \in S_{d m}(\Omega)
$$

Then $P^{d}$ is bounded, that is,

$$
\left\|\nabla P^{d} u^{n, 0}\right\|_{0} \leq\left\|\nabla u^{n, 0}\right\|_{0}, \quad \forall u^{n, 0} \in S_{d m}(\Omega) .
$$

Thus, by functional analysis theories (see [39]), there exists an extension $P^{h}: U \rightarrow$ $L^{2}\left(0, T ; S_{h m}(\Omega)\right)$ such that $\left.P^{h}\right|_{h m}(\Omega)=P^{d}: S_{h m}(\Omega) \rightarrow S_{d m}(\Omega)$ defined by

$$
\left(\nabla P^{h} u, \nabla w_{h}\right)=\left(\nabla u, \nabla w_{h}\right), \quad \forall w_{h} \in L^{2}\left(0, T ; S_{h m}(\Omega)\right),
$$

where $u \in U$. Due to (3.5), the projection $P^{h}$ is also bounded,

$$
\left\|\nabla\left(P^{h} u\right)\right\|_{0} \leq\|\nabla u\|_{0}, \quad \forall u \in U
$$

There is the following inequality (see $[24,26,27])$ :

$$
\left\|u-P^{h} u\right\|_{0} \leq C h\left\|\nabla\left(u-P^{h} u\right)\right\|_{0}, \quad \forall u \in U .
$$

Further, there are the following conclusions (see $[8,24,26,27])$.

Lemma 3.2 For every $d(1 \leq d \leq \kappa)$, the projection $P^{d}$ satisfies

$$
\frac{1}{M} \sum_{i=1}^{M}\left[\left\|u^{i, 0}-P^{d} u^{i, 0}\right\|_{0}^{2}+h^{2}\left\|\nabla\left(u^{i, 0}-P^{d} u_{h}^{i, 0}\right)\right\|_{0}^{2}\right] \leq C h^{2} \sum_{j=d+1}^{\kappa} \lambda_{j},
$$

where $u^{i, 0} \in S_{h m}(\Omega)(i=1,2, \ldots, M)$ are the solutions to (2.14). Further, if $u \in H^{r}(0, T$; $\left.H^{m+1}(\Omega)\right)$ is the solution to Problem II, then, for all $t \in[0, T]$, the projection $P^{h}$ satisfies the following error estimates:

$$
\left\|\partial_{t}^{r}\left[u(x, y, t)-P^{h} u(x, y, t)\right]\right\|_{s} \leq C h^{m+1-s}\left\|\partial_{t}^{r} u(x, y, t)\right\|_{m+1},
$$

where $\partial_{t}^{r}=\partial^{r} / \partial_{t}^{r}, r=0,1$, and $s=-1,0,1$. 
Thus, based on $S_{d m}(\Omega)$, the POD-based reduced-order TSCFE extrapolation iterative format for the $2 \mathrm{D}$ heat equations is established as follows.

Problem IV Find $u^{d k} \in S_{d m}(\Omega) \otimes S_{k l}(0, T)$ such that

$$
\begin{aligned}
& \left.u^{d k}(x, y, t)\right|_{J_{n}}=\sum_{j=0}^{l} \tilde{\ell}_{n, j}(t) \sum_{i=1}^{d}\left(\nabla \psi_{i}, \nabla u^{n, j}\right) \psi_{i}, \quad n=1,2, \ldots, M ; \\
& \sum_{j=1}^{l} m_{i j}\left(u_{d}^{n, j}, \psi_{d}\right)+k_{n} w_{i}\left(\nabla u_{d}^{n, i}, \psi_{d}\right) \\
& =\int_{J^{n}} \ell_{n, i}\left(f(x, y, t), \psi_{d}\right) \mathrm{d} t, \quad i=1,2, \ldots, l, \forall \psi_{d} \in S_{d m}(\Omega), \\
& \left.u^{d k}(x, y, t)\right|_{J_{n}}=\sum_{j=0}^{l} \tilde{\ell}_{n, j}(t) u_{d}^{n, j}(x, y), \quad n=M+1, M+2, \ldots, N,
\end{aligned}
$$

where $m_{i, j}=\int_{j^{n}} \tilde{\ell}_{n, j}^{\prime}(t) \ell_{n, i}(t) \mathrm{d} t(i=1,2, \ldots, l ; j=0,1, \ldots, l)$ and $u^{n, j}(n=1,2, \ldots, M ; j=$ $0,1, \ldots, l)$ are the solutions to $(2.14)$.

Remark 3.3 If $\Im_{h}$ is a uniformly regular triangulation, even though $S_{h m}(\Omega)$ is the spaces of piecewise linear polynomials, i.e., $m=1$, the number of total degrees of freedom for Problem III on each time level is $N_{h} \times l$ (where $N_{h}$ is the number of vertices of triangles in $\Im_{h}$ ). If $m=2$, the number of total degrees of freedom for Problem III on each time level is $4 N_{h} \times l$, while the number of total degrees of freedom for Problem IV on each time level is $d \times l\left(d \ll \kappa \leq M \ll N \ll N_{h}\right)$. For scientific engineering problems, the number $N_{h}$ of vertices of triangles in $\Im_{h}$ is more than tens of thousands, even more than a hundred million, while $d$ is only the number of the first few main eigenvalues so that it is very small (for example, in Section 5, $d=6$, while $N_{h}>2 \times 10^{2} \times 2 \times 10^{2}=4 \times 10^{4}$ ). Therefore, Problem IV is the POD-based reduced-order TSCFE extrapolation iterative format with very few degrees of freedom for the $2 \mathrm{D}$ heat equations. Especially, it has no repeating computations and uses the given solutions on the first fewer $M$ time steps for Problem III to extrapolate other $(n-M)$ solutions, which is completely different from existing reducedorder approaches (see, e.g., [14-35] etc.).

\section{Error analysis and algorithm implementation of POD-based reduced-order TSCFE extrapolation iterative format}

\subsection{Error estimates of solutions for Problem IV}

In the following, we employ classical TSCFE method to derived the error estimates of the POD-based reduced-order TSCFE solutions for Problem IV. We have the following main results for Problem IV.

Theorem 4.1 Under the hypotheses of Theorem 2.1, Problem IV has a unique solution $u^{d k} \in S_{d m}(\Omega) \otimes S_{k l}(0, T)$ such that

$$
\begin{aligned}
& \left\|\nabla u^{d k}\left(t_{n}\right)\right\|_{0} \leq C\left(\|f\|_{L^{2}\left(0, t_{n} ; H^{-1}\right)}+\left\|\nabla \varphi_{0}\right\|_{0}\right), \quad n=1,2, \ldots, M ; \\
& \left\|u_{t}^{h k}\right\|_{L^{2}\left(0, t_{n} ; H^{1}\right)}+\left\|\nabla u^{h k}\left(t_{n}\right)\right\|_{0} \\
& \quad \leq C\left(\|f\|_{L^{2}\left(0, t_{n} ; H^{-1}\right)}+\left\|\nabla \varphi_{0}\right\|_{0}\right), \quad n=M+1, M+2, \ldots, N,
\end{aligned}
$$


which show that the solutions to Problem IV are stable and continuously dependent of the initial value function $\varphi_{0}(x, y)$ and the source term $f(x, y, t)$. If $M^{2}=O(N)$, then we have the following error estimates between the solution $u(t)$ to Problem I and the solutions $u^{d k}$ to Problem IV:

$$
\begin{aligned}
& \left\|\left(u-u^{d k}\right)\left(x, y, t_{n}\right)\right\|_{0} \\
& \leq C\left[h^{m+1}\left(\|u\|_{H^{1}\left(0, t_{n} ; H^{m+1}\right)}+\left\|u\left(t_{n}\right)\right\|_{m+1}\right)+k^{l+1}\|u\|_{H^{l+1}\left(0, t_{n} ; H^{1}\right)}\right] \\
& \quad+C\left(k^{-1 / 2} h^{2} \sum_{i=d+1}^{\kappa} \lambda_{j}\right)^{1 / 2}, \quad n=1,2, \ldots, M ; \\
& \left\|\nabla\left(u\left(t_{n}\right)-u^{d k}\left(t_{n}\right)\right)\right\|_{1}+\left\|u^{h k}-u^{d k}\right\|_{H^{1}\left(t_{M}, t_{n} ; L^{2}\right)} \\
& \leq C\left[h^{m}\left(\left\|u_{t}\right\|_{L^{2}\left(0, t_{n} ; H^{m}\right)}+\left\|u\left(x, y, t_{n}\right)\right\|_{m+1}\right)\right. \\
& \left.\quad+k^{l}\|u\|_{H^{l+1}\left(0, t_{n} ; H^{2}\right)}\right], \quad n=M+1, M+2, \ldots, N .
\end{aligned}
$$

Proof The solutions of Problem IV on $J_{n}(n=1,2, \ldots, M)$ are obviously unique since they are obtained by projecting the solutions of (2.14) and (2.15), namely Problem III on $J_{n}$ $(n=1,2, \ldots, M)$ into POD basis. Moreover, (4.1) and (4.3) are immediately obtained from (3.6)-(3.7) and Theorem 2.1.

For $t \in J_{n}(n=M+1, M+2, \ldots, N)$, Problem IV has a unique solution by means of the same technique as the proof of Theorem 3.1 in [1] or the analogous method in [3]. Equations (3.11) and (3.12) are equivalently written as

$$
\int_{J_{n}}\left[\left(u_{t}^{d k}, \phi_{t}^{d}\right)+a\left(u^{d k}, \phi_{t}^{d}\right)\right] \mathrm{d} t=\int_{J_{n}}\left(f, \phi_{t}^{d}\right) \mathrm{d} t, \quad \forall \phi^{d} \in U_{d k}^{n}
$$

where $U_{d k}^{n}=S_{d m}(\Omega) \otimes S_{k l}\left(J_{n}\right)(n=M+1, M+2, \ldots, N)$ and $u^{d k}\left(x, y, t_{L}\right)=P^{d} u^{L, 0}(x, y)=$ $\sum_{j=1}^{d}\left(\nabla \psi_{j}, \nabla u^{L, 0}\right) \psi_{j}((x, y) \in \Omega)$. By subtracting (4.5) from Problem III taking $\phi=\phi^{d}$, we obtain the following system of error equations:

$$
\int_{J_{n}}\left[\left(u_{t}^{h k}-u_{t}^{d k}, \phi_{t}^{d}\right)+a\left(u^{h k}-u^{d k}, \phi_{t}^{d}\right)\right] \mathrm{d} t=0, \quad \forall \phi^{d} \in U_{d k}^{n}
$$

where $n=M+1, M+2, \ldots, N$ and $\left(u^{h k}-u^{d k}\right)\left(t_{M}\right)=\left(u^{h k}-P^{d} u^{h k}\right)\left(t_{M}\right)=u^{M, 0}(x, y)-$ $P^{d} u^{M, 0}(x, y)$.

Note that $J_{n}=\left[t_{n-1}, t_{n}\right]$. By using the system of error equations (4.6), (3.5), and the Hölder and Cauchy inequalities, we have

$$
\begin{aligned}
& \left\|u^{h k}-u^{d k}\right\|_{H^{1}\left(J_{n} ; L^{2}\right)}^{2}+\frac{1}{2}\left\|\nabla\left(u^{h k}-u^{d k}\right)\left(t_{n}\right)\right\|_{0}^{2}-\frac{1}{2}\left\|\nabla\left(u^{h k}-u^{d k}\right)\left(t_{n-1}\right)\right\|_{0}^{2} \\
& =\int_{J_{n}}\left[\left(u_{t}^{h k}-u_{t}^{d k}, u_{t}^{h k}-u_{t}^{d k}\right)+a\left(u^{h k}-u^{d k}, u_{t}^{h k}-u_{t}^{d k}\right)\right] \mathrm{d} t \\
& =\int_{J_{n}}\left[\left(u_{t}^{h k}-u_{t}^{d k}, u_{t}^{h k}-P^{d} u_{t}^{h k}\right)+a\left(u^{h k}-u^{d k}, u_{t}^{h k}-P^{d} u_{t}^{h k}\right)\right] \mathrm{d} t \\
& \quad+\int_{J_{n}}\left[\left(u_{t}^{h k}-u_{t}^{d k}, P^{d} u_{t}^{h k}-u_{t}^{d k}\right)+a\left(u^{h k}-u^{d k}, P^{d} u_{t}^{h k}-u_{t}^{d k}\right)\right] \mathrm{d} t
\end{aligned}
$$




$$
\begin{aligned}
= & \int_{J_{n}}\left[\left(u_{t}^{h k}-u_{t}^{d k}, u_{t}^{h k}-P^{d} u_{t}^{h k}\right)+\frac{1}{2} \frac{\mathrm{d}}{\mathrm{d} t} a\left(u^{h k}-P^{d} u^{h k}, u^{h k}-P^{d} u^{h k}\right)\right] \mathrm{d} t \\
\leq & \frac{1}{2}\left\|u^{h k}-u^{d k}\right\|_{H^{1}\left(J_{n} ; L^{2}\right)}^{2}+\frac{1}{2}\left\|u^{h k}-P^{d} u^{h k}\right\|_{H^{1}\left(J_{n} ; L^{2}\right)}^{2} \\
& +\frac{1}{2}\left\|\nabla\left(u^{h k}-P^{d} u^{h k}\right)\left(t_{n}\right)\right\|_{0}^{2}-\frac{1}{2}\left\|\nabla\left(u^{h k}-P^{d} u^{h k}\right)\left(t_{n-1}\right)\right\|_{0}^{2} .
\end{aligned}
$$

Note that $\left(u^{h k}-P^{d} u^{h k}\right)\left(t_{M}\right)=\left(u^{h k}-u^{d k}\right)\left(t_{M}\right)$. By simplifying the above inequality and summing it from $M+1$ to $n$, we obtain

$$
\begin{aligned}
& \left\|\nabla\left(u^{h k}-u^{d k}\right)\left(t_{n}\right)\right\|_{0}^{2}+\left\|u^{h k}-u^{d k}\right\|_{H^{1}\left(t_{M}, t_{n} ; L^{2}\right)}^{2} \\
& \quad \leq\left\|\nabla\left(u^{h k}-P^{d} u^{h k}\right)\left(t_{n}\right)\right\|_{0}^{2}+\left\|u^{h k}-P^{d} u^{h k}\right\|_{H^{1}\left(t_{M}, t_{n} ; L^{2}\right)}^{2}
\end{aligned}
$$

Moreover, by Theorem 2.1 and Lemma 3.2, we have

$$
\begin{aligned}
& \| \nabla\left(u^{h k}-P^{d} u^{h k}\right)\left(t_{n}\right) \|_{0} \\
& \leq\left\|\nabla\left(u^{h k}-u\right)\left(t_{n}\right)\right\|_{0}+\left\|\nabla\left(u-P^{h} u\right)\left(t_{n}\right)\right\|_{0}+\left\|\nabla\left(P^{h} u-P^{d} u^{h k}\right)\left(t_{n}\right)\right\|_{0} \\
& \leq\left\|\nabla\left(u-P^{h} u\right)\left(t_{n}\right)\right\|_{0}+2\left\|\nabla\left(u-u^{h k}\right)\left(t_{n}\right)\right\|_{0} \\
& \leq C\left[h^{m}\left(\|u\|_{H^{1}\left(0, t_{n} ; H^{m}\right)}+\left\|u\left(x, y, t_{n}\right)\right\|_{m+1}\right)+k^{l+1}\|u\|_{H^{l+1}\left(0, t_{n} ; H^{2}\right)}\right], \\
& n=M+1, M+2, \ldots, N \\
&\left\|u^{h k}-P^{d} u^{h k}\right\|_{H^{1}\left(t_{M}, t_{n} ; L^{2}\right)} \\
& \leq C\left\{k^{l}\left[\|u\|_{H^{l+1}\left(0, t_{n} ; L^{2}\right)}+\|u\|_{H^{l}\left(0, t_{n} ; H^{2}\right)}\right]+h^{m+1}\left\|u_{t}\right\|_{L^{2}\left(0, t_{n} ; H^{m+1}\right)}\right\}, \\
& \quad n=M+1, M+2, \ldots, N .
\end{aligned}
$$

Combining (4.7) with (4.8) and (4.9) yields

$$
\begin{aligned}
& \left\|\nabla\left(u^{h k}-u^{d k}\right)\left(t_{n}\right)\right\|_{0}+\left\|u^{h k}-u^{d k}\right\|_{H^{1}\left(t_{M}, t_{n} ; L^{2}\right)} \\
& \leq C\left[h^{m}\left(\|u\|_{H^{1}\left(0, t_{n} ; H^{m}\right)}+\left\|u\left(x, y, t_{n}\right)\right\|_{m+1}\right)\right. \\
& \left.\quad+k^{l}\left[\|u\|_{H^{l+1}\left(0, t_{n} ; L^{2}\right)}+\|u\|_{H^{l}\left(0, t_{n} ; H^{2}\right)}\right]\right], \quad n=M+1, M+2, \ldots, N .
\end{aligned}
$$

Combining (4.10) with Theorem 2.1 and (4.3) yields (4.4).

With Theorem 2.1 and by means of the analogous proof of Theorem 4.1, we easily obtain the following corollary.

Corollary 4.2 Under the hypotheses of Theorems 2.1 and 4.1, we have the following $L^{2}$-error estimates for the solutions $u^{d k}$ to Problem IV:

$$
\begin{aligned}
\left\|\left(u-u^{d k}\right)\left(x, y, t_{n}\right)\right\|_{0} \leq & C\left[h^{m+1}\left(\|u\|_{H^{1}\left(0, t_{n} ; H^{m+1}\right)}+\left\|u\left(t_{n}\right)\right\|_{m+1}\right)+k^{l+1}\|u\|_{H^{l+1}\left(0, t_{n} ; H^{1}\right)}\right] \\
& +C\left(k^{-1 / 2} h^{2} \sum_{i=d+1}^{\kappa} \lambda_{j}\right)^{1 / 2}, \quad n=1,2, \ldots, N
\end{aligned}
$$


Remark 4.3 Due to POD-based reduced-order and extrapolation for the classical TSCFE formulation Problem III, the errors of the solutions for the POD-based reduced-order TSCFE extrapolation iterative format Problem IV include more term $\left(k^{-1 / 2} h^{2} \sum_{i=d+1}^{\kappa} \lambda_{j}\right)^{1 / 2}$ than those for Problem III, but the degrees of freedom for Problem IV are far less than those of Problem III so that Problem IV can greatly lessen the truncation error accumulation in the computational process, alleviate the calculating load, save time-consuming calculations, and improve actual computational accuracy (see the example in Section 5). However, the factor $\left(k^{-1 / 2} h^{2} \sum_{i=d+1}^{\kappa} \lambda_{j}\right)^{1 / 2}$ in Theorem 4.1 and Corollary 4.2 can act as a suggestion to choose the number of POD bases, namely, it is only necessary to choose $d$ such that $\left(k^{-1 / 2} h^{2} \sum_{i=d+1}^{\kappa} \lambda_{j}\right)^{1 / 2}=O\left(k^{l}, h^{m}\right)$.

\subsection{Algorithm implementation of POD-based reduced-order TSCFE extrapolation iterative format}

Finding the solutions of the POD-based reduced-order TSCFE extrapolation iterative format for $2 \mathrm{D}$ heat equations consists of the following six steps.

Step 1 . For given initial value function $\varphi_{0}(x, y)$, source term $f(x, y, t)$, and the time step increment $k$ and the spatial grid measurement $h$, solving (2.14) at the first $M$ steps obtains the snapshots $u^{n, 0}(n=1,2, \ldots, M)$.

Step 2. Form the correlation matrix $\mathbf{A}=\left(A_{i j}\right)_{M \times M} \in R^{M \times M}$, where $A_{i j}=\left(\nabla u^{i, 0}, \nabla u^{j, 0}\right) / M$ and $(\cdot, \cdot)$ is $L^{2}$-inner product.

Step 3. Let $\mathbf{v}=\left(a_{1}, a_{2}, \ldots, a_{M}\right)^{T}$. Solving the eigenvalue problem $\mathbf{A v}=\lambda \mathbf{v}$ yields positive eigenvalues $\lambda_{1} \geq \lambda_{2} \geq \cdots \geq \lambda_{\kappa}>0\left(\kappa=\operatorname{dim}\left(\operatorname{span}\left\{u^{n, 0}(x, y) ; n=1,2, \ldots, M\right\}\right)\right)$ and the corresponding eigenvectors $\mathbf{v}^{j}=\left(a_{1}^{j}, a_{2}^{j}, \ldots, a_{M}^{j}\right)^{T}(j=1,2, \ldots, \kappa)$.

Step 4. For the error $\delta=O\left(k^{l}, h^{m}\right)$ needed, determine the number $d$ of POD basis such that $k^{-1 / 2} h^{2} \sum_{j=d+1}^{\kappa} \lambda_{j} \leq \delta^{2}$.

Step 5. Generate POD basis $\psi_{i}=\sum_{j=1}^{M} a_{j}^{i} u^{j, 0}(x, y) / \sqrt{M \lambda_{i}}(j=1,2, \ldots, d)$. Let $S_{d m}(\Omega)=$ $\operatorname{span}\left\{\psi_{1}, \psi_{2}, \ldots, \psi_{d}\right\}$. Solving Problem IV with $d$ degrees of freedom obtains $u^{d k}(x, y, t)$.

Step 6. If $\left\|\nabla\left(u_{d}^{n-1,0}-u_{d}^{n, 0}\right)\right\|_{0} \geq\left\|\nabla\left(u_{d}^{n, 0}-u_{d}^{n+1,0}\right)\right\|_{0}(n=M, M+1, \ldots, N-1)$, then $u^{d k}$ are the solutions for Problem IV satisfying accuracy needed. Else, namely, if $\| \nabla\left(u_{d}^{n-1,0}-\right.$ $\left.u_{d}^{n, 0}\right)\left\|_{0}<\right\| \nabla\left(u_{d}^{n, 0}-u_{d}^{n+1,0}\right) \|_{0}(n=M, M+1, \ldots, N-1)$, let $u^{i, 0}=u^{n-M-i}(i=1,2, \ldots, M)$ and return to Step 2.

\section{A numerical example}

In this section, we present a numerical example showing the advantage of the POD-based reduced-order TSCFE extrapolation iterative format for $2 \mathrm{D}$ heat equations.

Its computational domain is irregular and consists of a set $\bar{\Omega}=([0,2] \times[0,2]) \cup$ $([2,2.03] \times[0.65,1.3]) \mathrm{cm}^{2}$. The triangularization $\Im_{h}$ for computational domain $\bar{\Omega}$ adopts local refining meshes such that the scale of meshes on $[2,2.03] \times[0.65,1.3]$ and nearby $(2, y)(0 \leq y \leq 2)$ are one third of meshes nearby $(0, y)(0 \leq y \leq 2)$. But $h=\sqrt{2} \times 10^{-2}$, whose nodes $N_{h}>4 \times 10^{4}$. We take the time step increment as $k=10^{-2} \mathrm{~s}$ (second). Subspaces $S_{h m}(\Omega)$ and $S_{k l}(0, T)$ are taken as piecewise second polynomial spaces, namely, $m=2$ and $l=2$, respectively. The source term is taken as $f(x, y, t)=0$, the initial and boundary valve functions are taken as follows, for $0 \leq t \leq T$ :

$$
\varphi_{0}(x, y)= \begin{cases}1.5, & \text { if }(x, y) \in[2,2.03] \times[0.65,1.3], \\ 1.0, & \text { if }(x, y) \in[2,2] \times([0,0.5] \cup[1.5,2]), \\ 0.0, & \text { others }\end{cases}
$$


$\varphi_{1}(x, y)=0$, and the boundary value function as follows:

$$
\varphi(x, y, t)= \begin{cases}\varphi_{0}(x, y), & (x, y) \in \partial \Omega, t=0, \\ u_{h}^{n-1}(x, y, t), & (x, y) \in \partial \Omega, t=t_{n}, n=1,2, \ldots, n .\end{cases}
$$

We first take the first $M=20$ solutions $u^{n, 0}(x, y)=u\left(x, y, t_{n}\right)$ for the classical TSCFE formulation, namely, Problem III at time partition points $t_{n}(n=1,2, \ldots, 20)$ as snapshots. Then we find the eigenvalues $\lambda_{1} \geq \lambda_{2} \geq \cdots \geq \lambda_{20} \geq 0$ and the corresponding eigenvectors $\mathbf{v}^{j}=\left(a_{1}^{j}, a_{2}^{j}, \ldots, a_{20}^{j}\right)^{T}(j=1,2, \ldots, 20)$ for the matrix $\mathbf{A}=\left(A_{i j}\right)_{20 \times 20}$, where $A_{i j}=$ $\left(\nabla u^{i, 0}, \nabla u^{j, 0}\right) / 20$. It is shown by computing that error factor $\left(k^{-1 / 2} h^{2} \sum_{i=7}^{20} \lambda_{j}\right)^{1 / 2} \leq 4 \times 10^{-6}$. Thus, it is only necessary to take the most main six POD bases. According to 6 steps in Section 4.2 without renewing POD basis, the POD-based reduced-order TSCFE extrapolation iterative format are still convergent, whose solution at $t=10$ is found and depicted on the top chart in Figure 1. The numerical solution $u_{h}^{n}$ obtained with classical Problem III when $n=1,000$ (i.e., $t=10$ ) is depicted graphically on the bottom chart in Figure 1 . The two charts in Figure 1 exhibit a quasi-identical similarity. Although the errors of the PODbased reduced-order TSCFE solutions on the starting time span are slightly larger than those of the classical TSCFE solutions, since the POD-based reduced-order TSCFE ex-
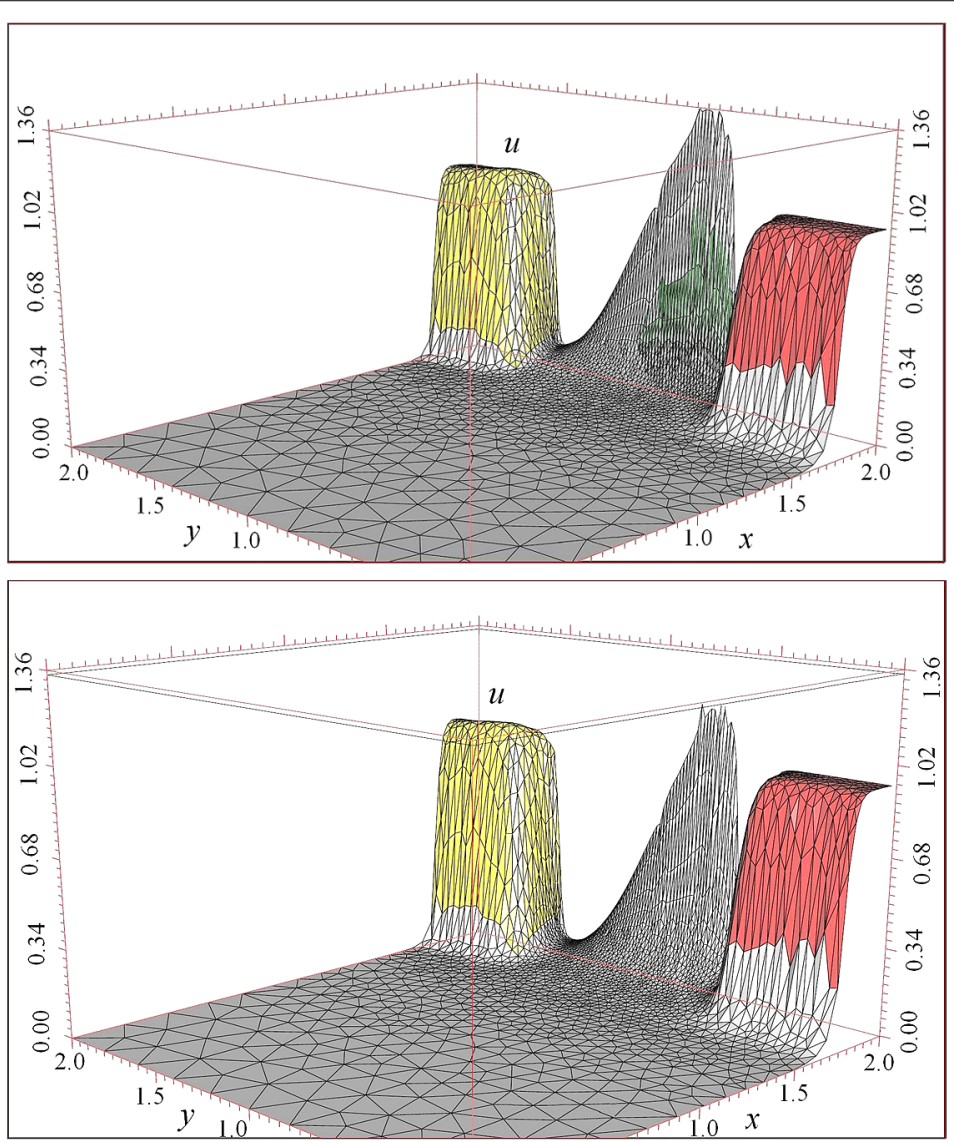

Figure 1 Classical TSCFE solutions $u_{d}^{n}$ and the reduced-order TSCFE solution $u_{d}^{n}$. The top chart is the reduced-order TSCFE solution $u_{d}^{n}$ when $d=6$ at $n=1,000(t=10)$. The bottom chart is the classical TSCFE solution $u_{h}^{n}$ at $n=1,000(t=10)$. 


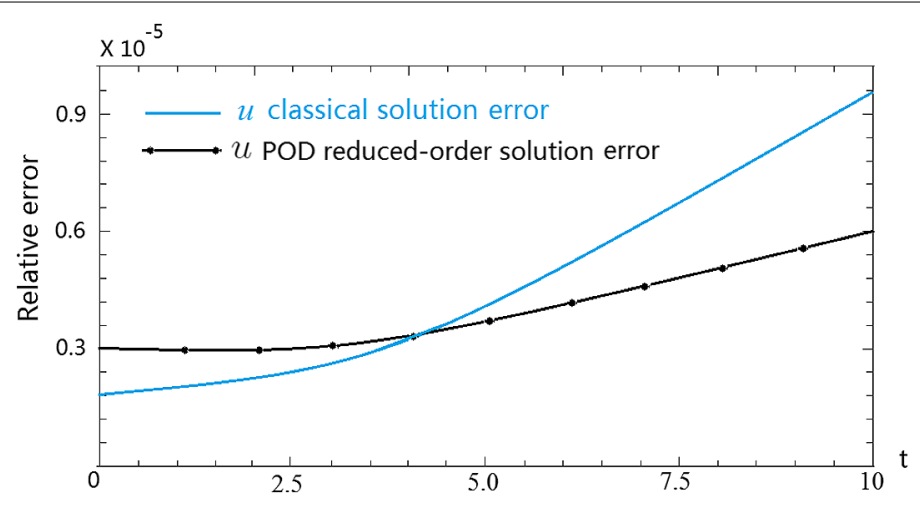

Figure 2 The relative errors of the classical TSCFE solution and the POD-based reduced-order TSCFE solution with six POD bases on $0 \leq t \leq 10$.

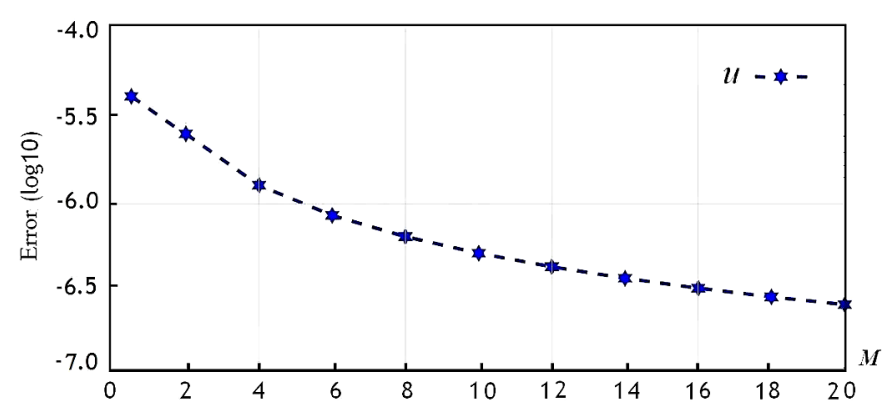

Figure 3 The error $(\log 10)$ chart between the classical TSCFE solution and the POD-based reduced-order TSCFE solutions with different number of 20 POD bases at $t=10$.

trapolation iterative format Problem IV on each time level only includes $2 \times 6$ degrees of freedom and the classical TSCFE formulation Problem III has more than $2 \times 16 \times 10^{4}$ degrees of freedom, namely, the degrees of freedom for the POD-based reduced-order TSCFE extrapolation iterative format are far fewer than those for the classical TSCFE formulation Problem III so that it could greatly lessen the truncation error accumulation in the computational process, alleviate the calculating load, save the consuming time of calculations, and improve the actual computational accuracy; therefore, after some time span, the numerical errors of the POD-based reduced-order TSCFE extrapolation iterative format are fewer than those of the classical TSCFE formulation (see Figure 2).

Figure 3 shows the errors between solutions obtained from the POD-based reducedorder TSCFE extrapolation iterative format with different number of POD bases and solutions obtained from the classical TSCFE formulation at $t=10$. It is shown that the numerical computing results are consistent with those obtained for the theoretical case because the theoretical and numerical errors are all $O\left(10^{-6}\right)$.

Further, by comparing the classical TSCFE formulation with the POD-based reducedorder TSCFE extrapolation iterative format with six POD bases implementing the numerical simulations at $t=10$, it is found that the classical TSCFE formulation includes more than $2 \times 16 \times 10^{4}$ unknown quantities (since the subspaces $S_{h m}(\Omega)$ and $S_{k l}(0, T)$ are taken as piecewise second polynomial spaces) on each time level and the required computing time is 240 minutes, while the POD-based reduced-order TSCFE extrapolation iterative 
format with six POD bases does only include six unknown quantities on each time level and the corresponding computing time is less than 60 seconds, namely, the computing time of the classical TSCFE formulation is 240 times higher than that of the POD-based reduced-order TSCFE extrapolation iterative format with six POD bases. Thus, the PODbased reduced-order TSCFE extrapolation iterative format can greatly save the calculating time and alleviate the computing load so that it could greatly lessen the truncation error accumulation in the computational process. It is also shown that finding the numerical solutions for 2D heat equations by means of the POD-based reduced-order TSCFE extrapolation iterative format is computationally very effective and feasible.

\section{Conclusions and discussions}

In this article, the POD-based reduced-order TSCFE extrapolation iterative format for 2D heat equations has been established, the error estimates of the POD-based reducedorder TSCFE solutions and the algorithm implementation of the POD-based reducedorder TSCFE extrapolation iterative format have been provided. A numerical example has been used to validate that the results of the numerical computation are consistent with the theoretical conclusions. Further, it has shown that the POD-based reduced-order TSCFE extrapolation iterative format can greatly save the calculating time and alleviate the computing load so that it could greatly lessen the truncation error accumulation in the computational process. Moreover, it is shown that the POD-based reduced-order TSCFE extrapolation iterative format is computationally very effective and feasible for finding the numerical solutions to $2 \mathrm{D}$ heat equations.

Though some POD-based reduced-order models for 2D parabolic equations have been established (see [15, 19, 23, 27, 31]), the POD-based reduced-order TSCFE extrapolation iterative format here is completely different from those mentioned above because the TSCFE method is different from the usual Galerkin method, the FE method, the finite volume method, and the finite difference scheme. Especially, the POD-based reduced-order TSCFE extrapolation iterative format only employs the first few given classical TSCFE solutions on the very short time span $\left[0, T_{0}\right]\left(T_{0} \ll T\right)$ as snapshots to form the POD basis and seek the numerical solutions on the total time span $[0, T]$. It is an improvement and innovation of the existing methods (see, e.g., [14-35] etc.).

Future research work in this area will aim to extend the POD-based reduced-order TSCFE extrapolation iterative format, applying it to more TDPDEs such as the nonlinear Schrödinger equation, integral differential equations, convection-diffusion equations, nonlinear sine-Gordon equation, Sobolev equations, solute transport equations, and viscoelastic equations.

Competing interests

The author declares that he has no competing interests.

\section{Acknowledgements}

This research was supported by National Science Foundation of China grant 11271127 and Science Research Project of Guizhou Province Education Department grant QJHKYZ[2013]207.

Received: 19 October 2014 Accepted: 23 March 2015 Published online: 04 April 2015

References

1. Aziz, AK, Monk, P: Continuous finite elements in space and time for the heat equation. Math. Comput. 52, 255-274 (1989)

2. Karakashia, O, Makkridakis, C: A time-space finite element method for nonlinear Schrödinger equation: the continuous Galerkin method. Math. Comput. 97(222), 479-499 (1998) 
3. Li, H, Liu, RX: The space-time finite element method for parabolic problems. Appl. Math. Mech. 22(6), 687-700 (2001)

4. Bales, L, Lasiecka, I: Continuous finite elements in space and time for the nonhomogeneous wave equation. Comput. Math. Appl. 27(3), 91-102 (1994)

5. French, DA, Peterson, TE: A continuous space-time finite element method for the wave equation. Math. Comput. 65(214), 491-506 (1996)

6. Liu, Y, Li, H, He, S: Mixed time discontinuous space-time finite element method for convection diffusion equations. Appl. Math. Mech. 29(12), 1579-1586 (2008)

7. French, DA: A space-time finite element method for the wave equation. Comput. Methods Appl. Mech. Eng. 107, 145-157 (1993)

8. Luo, ZD: The Foundations and Applications of Mixed Finite Element Methods. Chinese Science Press, Beijing (2006) (in Chinese)

9. Thomée, V: Galerkin Finite Element Methods for Parabolic Problems, 2nd edn. Springer, Berlin (2003)

10. Thomée, V: Negative norm estimates and superconvergence in Galerkin methods for parabolic problems. Math Comput. 34, 93-113 (1980)

11. Holmes, P, Lumley, JL, Berkooz, G: Turbulence, Coherent Structures, Dynamical Systems and Symmetry. Cambridge University Press, Cambridge (1996)

12. Fukunaga, K: Introduction to Statistical Recognition. Academic Press, New York (1990)

13. Jolliffe, IT: Principal Component Analysis. Springer, Berlin (2002)

14. Ly, HV, Tran, HT: Proper orthogonal decomposition for flow calculations and optimal control in a horizontal CVD reactor. Q. Appl. Math. 60, 631-656 (2002)

15. Kunisch, K, Volkwein, S: Galerkin proper orthogonal decomposition methods for parabolic problems. Numer. Math. 90, 117-148 (2001)

16. Kunisch, K, Volkwein, S: Galerkin proper orthogonal decomposition methods for a general equation in fluid dynamics. SIAM J. Numer. Anal. 40, 492-515 (2002)

17. Kunisch, K, Volkwein, S: Control of Burgers' equation by a reduced order approach using proper orthogonal decomposition. J. Optim. Theory Appl. 102, 345-371 (1999)

18. AhIman, D, Södelundon, F, Jackson, J, Kurdila, A, Shyy, W: Proper orthogonal decomposition for time-dependent lid-driven cavity flows. Numer. Heat Transf., Part B, Fundam. 42, 285-306 (2002)

19. Luo, ZD, Chen, J, Xie, ZH, An, J, Sun, P: A reduced second-order time accurate finite element formulation based on POD for parabolic equations. Sci. Sin., Math. 41(5), 447-460 (2011) (in Chinese)

20. Luo, ZD, Li, H, Zhou, YJ, Huang, XM: A reduced FVE formulation based on POD method and error analysis for two-dimensional viscoelastic problem. J. Math. Anal. Appl. 385, 371-383 (2012)

21. Luo, ZD, Li, H, Zhou, YJ, Xie, ZH: A reduced finite element formulation and error estimates based on POD method for two-dimensional solute transport problems. J. Math. Anal. Appl. 385, 371-383 (2012)

22. Luo, ZD, Du, J, Xie, ZH, Guo, Y: A reduced stabilized mixed finite element formulation based on proper orthogonal decomposition for the non-stationary Navier-Stokes equations. Int. J. Numer. Methods Eng. 88(1), 31-46 (2011)

23. Luo, ZD, Xie, ZH, Shang, YQ, Chen, J: A reduced finite volume element formulation and numerical simulations based on POD for parabolic equations. J. Comput. Appl. Math. 235(8), 2098-2111 (2011)

24. Luo, ZD, Xie, ZH, Chen, J: A reduced MFE formulation based on POD for the non-stationary conduction-convection problems. Acta Math. Sci. 31(5), 1765-1785 (2011)

25. Li, HR, Luo, ZD, Chen, J: Numerical simulation based on proper orthogonal decomposition for two-dimensional solute transport problems. Appl. Math. Model. 35(5), 2489-2498 (2011)

26. Luo, ZD, Zhou, YJ, Yang, XZ: A reduced finite element formulation based on proper orthogonal decomposition for Burgers equation. Appl. Numer. Math. 59(8), 1933-1946 (2009)

27. Luo, ZD, Chen, J, Sun, P, Yang, XZ: Finite element formulation based on proper orthogonal decomposition for parabolic equations. Sci. Sin., Math. 52(3), 587-596 (2009)

28. Luo, ZD, Chen, J, Navon, IM, Yang, XZ: Mixed finite element formulation and error estimates based on proper orthogonal decomposition for the non-stationary Navier-Stokes equations. SIAM J. Numer. Anal. 47(1), 1-19 (2008)

29. Luo, ZD, Chen, J, Navon, IM, Zhu, J: An optimizing reduced PLSMFE formulation for non-stationary conduction-convection problems. Int. J. Numer. Methods Fluids 60(4), 409-436 (2009)

30. Luo, ZD, Zhu, J, Wang, RW, Navon, IM: Proper orthogonal decomposition approach and error estimation of mixed finite element methods for the tropical Pacific Ocean reduced gravity model. Comput. Methods Appl. Mech. Eng 196(41-44), 4184-4195 (2007)

31. Luo, ZD, Li, H, Shang, YQ, Fang, Z: A LSMFE formulation based on proper orthogonal decomposition for parabolic equations. Finite Elem. Anal. Des. 60, 1-12 (2012)

32. Bergmann, M, Bruneau, CH, lollo, A: Enablers for robust POD models. J. Comput. Phys. 228(2), 516-538 (2009)

33. Deane, AE, Kevrekidis, IG, Karniadakis, GE, Orsag, SA: Low-dimensional models for complex geometry flows: application to grooved channels and circular cylinder. Phys. Fluids A 3(10), 2337-2354 (1991)

34. Ma, X, Karniadakis, GE: A low-dimensional model for simulating three-dimensional cylinder flow. J. Fluid Mech. 458, $181-190$ (2002)

35. Wang, Z, Akhtar, I, Borggaard, J, lliescu, T: Two-level discretizations of non-linear closure models for proper orthogonal decomposition. J. Comput. Phys. 230, 126-146 (2011)

36. Adams, RA: Sobolev Spaces. Academic Press, New York (1975)

37. Brezzi, F, Fortin, M: Mixed and Hybrid Finite Element Methods. Springer, New York (1991)

38. Ciarlet, PG: The Finite Element Method for Elliptic Problems. North-Holland, Amsterdam (1978)

39. Rudin, W: Functional Analysis, 2nd edn. McGraw-Hill, New York (1973) 